OPEN ACCESS

Edited by:

Ihtisham Bukhari,

Fifth Affiliated Hospital of Zhengzhou

University, China

Reviewed by:

Jianjun Dong,

Shandong University, China

Mohammed Amir Husain,

Université de Sherbrooke,

Canada

${ }^{*}$ Correspondence:

Zehua Song

song.zehua@outlook.com

Specialty section: This article was submitted to

Clinical Diabetes,

a section of the journal

Frontiers in Endocrinology

Received: 03 January 2022

Accepted: 27 January 2022

Published: 24 February 2022

Citation:

Zhen D, Liu J, Zhang XD and Song Z (2022) Kynurenic Acid Acts as a Signaling Molecule Regulating Energy Expenditure and Is Closely Associated With Metabolic Diseases.

Front. Endocrinol. 13:847611. doi: 10.3389/fendo.2022.847611

\section{Kynurenic Acid Acts as a Signaling Molecule Regulating Energy Expenditure and Is Closely Associated With Metabolic Diseases}

\author{
Delong Zhen ${ }^{2}$, Junjun Liu ${ }^{2}$, Xu Dong Zhang ${ }^{1}$ and Zehua Song ${ }^{1,3 *}$ \\ 1 Translational Research Institute, Henan Provincial People's Hospital and People's Hospital of Zhengzhou University, \\ Academy of Medical Science, Zhengzhou University, Zhengzhou, China, 2 Shandong Institute of Endocrine and Metabolic \\ Diseases, Shandong First Medical University \& Shandong Academy of Medical Sciences, Jinan, China, ${ }^{3}$ ENNOVA Institute of \\ Life Science and Technology, ENN Group, Langfang, China
}

Kynurenic acid (KYNA) is an important bio-active product of tryptophan metabolism. In addition to its well-known neuroprotective effects on mental health disorders, it has been proposed as a bio-marker for such metabolic diseases as atherosclerosis and diabetes. Emerging evidence suggests that KYNA acts as a signaling molecule controlling the networks involved in the balance of energy store and expenditure through GPR35 and AMPK signaling pathway. KYNA plays an important role in the pathogenesis and development of several endocrine and metabolic diseases. Exercise training promotes KYNA production in skeletal muscles and increases thermogenesis in the long term and limits weight gain, insulin resistance and inflammation. Additionally, KYNA is also present in breast milk and may act as an anti-obesity agent in infants. Although we are far from fully understanding the role of KYNA in our body, administration of KYNA, enzyme inhibitors or metabolites may serve as a potential therapeutic strategy for treating metabolic diseases. The present review provides a perspective on the current knowledge regarding the biological effects of KYNA in metabolic diseases and perinatal nutrition.

Keywords: kynurenic acid, inflammation, physical exercise, perinatal nutrition, metabolic diseases

\section{INTRODUCTION}

Kynurenic acid (KYNA) is one of the metabolites of tryptophan catabolism formed via the kynurenine pathway. It is first known for its neuro-protective effect as it is the only known broad-spectrum endogenous antagonist for ionotropic glutamate receptors. As such, a large number of studies have been carried out to investigate the role of KYNA in the physio-pathology of central nervous system (CNS) such as depression, Alzheimer's diseases and schizophrenia in the past two decades. In addition to such neuronal contributions, KYNA can also be found with higher concentration in urine, pancreatic mucus, serum and breast milk than in cerebrospinal fluid (1). It has been subsequently found to be involved in immune (2) and digestive system (1) in the periphery. Increasing reports have concentrated on the role of KYNA outside the CNS.

More recently, evidence suggests that physical exercise may also influence this pathway and KYNA has emerged as a signaling molecule for energy homeostasis in peripheral tissues. Some metabolomic and 
epidemiological studies have proposed that KYNA can serve as an early bio-marker for diabetes and some other metabolic diseases (3, 4). Furthermore, in some animal studies, KYNA has been considered as a significant protector against such metabolic diseases as obesity and nonalcoholic fatty liver disease (NAFLD) $(5,6)$. This essay summarizes recent advances of how KYNA is involved in the regulation of energy homeostasis of peripheral tissue and its potential role in the onset and progression of such metabolic diseases as obesity and diabetes.

\section{THE ENDOGENOUS PRODUCTION OF KYNA IN PERIPHERAL TISSUES}

The endogenous production of KYNA in human brain has been well studied over the past few decades because of its neuroprotective effects. However, increasing evidence showed that KYNA can also be found, even in higher concentration, in many body fluids in humans such serum (7), saliva (8), bile (9) and breast milk (10). In humans and rodents, the production of KYNA has been described in a number of peripheral tissues such as muscle (11), liver (12), kidney (12), pancreas $(9,13)$, endothelial cells (14) and immune cells (15) under physiological conditions. KYNA is produced mainly through the side branch of tryptophan/kynurenine pathway. Approximately 95\% of tryptophan is metabolized through kynurenine pathway, and about $0.3 \%$ is converted to KYNA (10 $\mu \mathrm{mol} /$ day) (16).

In this catabolic pathway, the first step is catalyzed by the enzymes indol-2,3-dioxygenase (IDO) and tryptophan-2,3dioxygenase (TDO) to generate $\mathrm{N}$-formyl-L-kynurenine, an unstable compound which is rapidly converted to L-kynurenine (L-KYN) by ubiquitous aryl formamidase (AFMID). TDO is mainly expressed in the liver and has a $K_{m}$ value of $190 \mu \mathrm{M}$ for TRP, which ensures that TDO is active to convert TRP to L-KYN at higher than physiological concentrations (about $80 \mu \mathrm{M})(17,18)$. Another limiting enzyme, IDO1, is expressed under the induction of proinflammatory cytokines (19). IDO1 has a $K_{m}$ value of $20 \mu \mathrm{M}$ for tryptophan, which is much lower than TDO (17). IDO2, a newly discovered enzyme, has some homology to IDO1, but its $K_{m}$ value for TRP is much higher than that of IDO1 and TDO, and has little effect on the production of TRP downstream metabolites (20). After this step, L-KYN can be either converted to nicotinamide by a series of reactions or enter into a side branch to produce KYNA. KYNA is supposed to be a final catabolic product of this side branch of kynurenine pathway because no further metabolite is reported in mammals (but can be further catabolized by intestinal flora).

The KYNA branch of the kynurenine pathway is mainly regulated by the activity of kynurenine aminotransferase (KYAT, EC 2.6.1.7), whose abbreviation is recently updated from KAT to KYAT. Four different isozymes of KYAT (KYAT1-4) are identified in mammalian cells. They are all members of the pyridoxal-5'-phosphate-dependent enzyme family and require an $\alpha$-ketoacid as the amino group acceptors.

KYAT1 catalyzes the transamination of L-KYN to form KYNA and its $K_{m}$ for L-KYN is around $4.7 \mathrm{mM}$ (21). It should be noted that KYAT1 possesses broad amino acid specificity and also catalyzes the transamination such as glutamine to $\alpha$ ketoglutaramate, thus it is also known as glutamine transaminase K (GTK, EC 2.6.1.15). Leucine, glutamate, methionine also seem to be the preferred substrates of human KYAT1 (21). But, to date, its most biologically significant product is KYNA. KYAT1 also possesses cysteine S-conjugate beta-lyases (CCBL1) activity and catalyzes the beta-elimination of cysteine S-conjugates to generate pyruvate and thioacylating fragments. This activity plays an important role in the bio-activation of cysteine S-conjugates found in garlic and onion (22) and in the toxification of some toxins like 5-S-l-cysteinyldopamine. Competitive inhibition test suggests KYAT1 has two active sites, one for KYAT and the other for GTK. The active sites of KYAT and CCBL1 may be the same (23). KYAT1 is both cytosolic and mitochondrial because it exists two different mRNA variants coding for proteins with and without mitochondria targeting sequence. In rats, KYAT1 mRNA can be detected in most of tissues such as small intestine, pancreas, lung, liver, heart, kidney, brain, muscle, testis, ovary $(23,24)$. In brain, the activity of KYAT1 is critical to the formation of KYNA, its activity is associated with schizophrenia (25). A missense mutation in KYAT1 was identified in spontaneously hypertensive rats. This mutation led to abnormally low KYNA levels in the area of central nervous system that controls blood pressure (26). Although the role of KYAT1 in brain has mostly been discussed, its expression is much higher in livers and kidneys than in brains (27). Its role in peripheral tissues did not receive much attention until the discovery of the immunomodulator and metabolic effect of KYNA.

KYAT2, the second isoform of the KYAT family, also possesses a broad-spectrum transamination activity with no S-conjugate betalyases activity. It is also known as alpha-aminoadipate aminotransferase (AADAT). Similar to KYAT1, its $K_{m}$ for L-KYN is around $4.7 \mathrm{mM}(28)$. KYAT2 can be detected in most tissues, but it is not detectable in murine skeletal muscle (11). KYAT2 appears to play a more important role in rat's brain because KYAT2 is highly expressed in astrocytes than other KYATs and KYNA is reported to be predominantly generated by it in the brain $(29,30)$.

Among the four KYATs, KYAT3 shares similar sequence and expression pattern to KYAT1 and is also known as glutamine transaminase L (GTL) and CCBL2 (EC 4.4.1.13). Likewise, KYAT3 is a multifunctional aminotransferase and catalyzes glutamine, methionine, phenylalanine, tyrosine and cysteine as transamination subtract, although it displayed no activity toward leucine (31). Its expression is much higher in kidney, liver and neuroendocrine tissues than in brain $(27,32)$.

KYAT4 is the last discovered KYAT. In fact, it is better known as mitochondrial glutamic-oxaloacetictransaminase 2 (EC 2.6.1.1), an essential player in the malate-aspartate shuttle in mitochondria and in the synthesis of glutamate (33). It is highly expressed in most tissues and organs because malate-aspartate shuttle is a general feature of cells with functional mitochondria, except for white adipose tissue (34).

Kynurenine 3-monooxygenase (KMO; EC 1.14.13.9) is an important regulator of KYNA synthesis as it is a kynurenineconsuming enzyme competing with KYAT for substrate. KMO is an NADPH-dependent flavin monooxygenase located in the outer membrane of mitochondria. It catalyzes the conversion of 
kynurenine to 3-hydroxykynurenine, a cytotoxic metabolite involved in the generation of ROS and activation of inflammatory response. KMO is widely expressed in our body, for it has been discovered in liver, kidney, pancreas, brain, macrophages, and monocytes $(3,13,35,36)$, with the highest $\mathrm{KMO}$ levels found in liver and kidney. Moreover, the activity of KMO in liver and kidney decreases significantly with aging (37).

KMO possesses higher affinity to kynurenine (7-14 $\mu \mathrm{M}$ for human $\mathrm{KMO}(38,39), 15-16 \mu \mathrm{M}$ for rodent (40) than all four types of KYATs. KMO inhibition by pharmacological inhibitor significantly increases KYNA levels (41). Also, in KMO knockout mice, the level of KYN and KYNA was significantly increased in the periphery (42). The characteristics of enzymes related to KYNA metabolism are summarized in Table $\mathbf{1}$.

Under some physiological or pathological conditions, KYNA can be produced from indole-3-Pyruvic Acid or from kynurenine by scavenging free radicals. These pathways represent alternative routes of KYNA production. Although the contributions of these alternative routes remain unclear, these could be very important in such metabolic diseases as obesity and diabetes, because these diseases share common factors such as oxidative stress and inflammation. This information has been reviewed by Ramos-Chávez et al. (47).

\section{THE TRANSPORT OF KYNA}

KYNA is able to cross the plasma membrane through organic anion transporters 1 and 3 (OAT1/SLC22A6 and OAT3/SLC22A8) (48). The proximal tubule of the kidney, where OATs are found, is not simply for renal elimination of KYNA and it also senses tryptophan metabolites levels and responds to changes in their intracellular abundance (49). It remains to be further investigated whether there exists an exocytosis gated KYNA secretion.

While KYNA does not easily cross the blood-brain barrier, KYNA synthesized by brain cannot be directly exported to the periphery and vice versa (50). However, since its precursor L-KYN can cross the blood-brain barrier (51), KYNA can be synthesized by KYAT in situ using transported L-KYN in central nervous system under certain conditions (51). Moreover, a recent study showed that in Caenorhabditis elegans, an ortholog of the human LAT1 transporter, AAT-1, imports L-KYN into sites of KYNA production (52). Another study showed that five amino acids, including leucine, isoleucine, methionine, phenylalanine and tyrosine, act as LAT substrates and

TABLE 1 | The characteristics of enzyme related to KYNA metabolism.

\begin{tabular}{lclc}
\hline Enzyme & $\boldsymbol{K}_{\boldsymbol{m}}$ & Substrate & References \\
\hline TPH1 & $8 \mu \mathrm{M}$ & Trp & $(43)$ \\
TPH2 & $41.3 \mu \mathrm{M}$ & Trp & $(44)$ \\
IDO1 & $20 \mu \mathrm{M}$ & Trp & $(45)$ \\
TDO2 & $190 \mu \mathrm{M}$ & Trp & $(17)$ \\
KMO & $7-16 \mu \mathrm{M}$ & $\mathrm{KYN}$ & $(38-40)$ \\
Kase & $493 \mu \mathrm{M}$ & $\mathrm{KYN}$ & $(46)$ \\
KYAT1 & $4700 \mu \mathrm{M}$ & $\mathrm{KYN}$ & $(21)$ \\
KYAT2 & $4700 \mu \mathrm{M}$ & $\mathrm{KYN}$ & $(28)$ \\
KYAT3 & $1500 \mu \mathrm{M}$ & $\mathrm{KYN}$ & $(31)$
\end{tabular}

inhibit brain KYNA synthesis by blocking L-KYN transport (53). Similar to this transport mechanism in brain, the uptake of KYNA in T cells can be mediated by the uptake of L-KYN via L-amino acid transporter SLC7A5 (54).

\section{LINKS BETWEEN KYNA AND COMMON METABOLIC DISEASES}

\section{Inflammation, the First Link Between KYNA and Metabolic Diseases}

The production of KYNA is directly correlated to inflammation as KYNA acts as an important immune regulated during inflammation response. KYNA inhibits TNF- $\alpha$ at transcriptional level and suppresses the secretion of TNF- $\alpha$ in mononuclear cells and in CD14 ${ }^{+}$monocytes (55). Oral administration of KYNA decreases the activity of the peripheral blood leukocytes in mice (56).

The immune response signaling pathway and metabolic regulation signaling pathway, especially insulin signaling pathway, are highly integrated, because organism would need to redistribute its energy resources during the activation of immune response (57). Chronic inflammation is activated in overweight individuals as a consequence of adipose expansion. Recent insights suggest that it may play an indispensable role in the over-nutrition induced insulin resistance (58). During the past two decades, it became clear that nutrient excess and activation of the innate immune system are highly associated in most organs such as adipose tissues, liver, gut, muscle, and islets (58). Low-grade chronic inflammation or metabolically triggered inflammation is considered as a fundamental characteristic of metabolic diseases particularly in the context of obesity and type 2 diabetes. Targeting inflammation has been suggested as an important strategy to prevent and control these diseases (58).

Numbers of studies suggest that KYNA is produced during inflammation and it has been shown to mediate various immunomodulatory effects under inflammatory conditions (2). Since inflammation is one of the main factors in many metabolic diseases, it can be foreseen that KYNA may also play an important regulatory role in the metabolic diseases.

Increased tryptophan/kynurenine metabolite levels are frequently observed in overweight individuals (3). An increased serum KYNA level can be found in Zucker fatty rats (59), and in HFD fed LDL receptor knockout mice (60). Clinically, serum KYNA has been found positively correlated with BMI in overweight individuals $(3,61)$. The elevation of serum KYNA concentrations is closely associated with the activation of immune cells as increased IDO1 activity in macrophages and increased serum KYNA levels have been reported in obese animal models. The increased KYNA levels may result from an up-regulated biosynthesis in the omental adipose tissue (but not in subcutaneous adipose tissue), as the expression levels of IDO1, KYAT1 and KYAT3 were significantly higher in overweight individuals than in lean individuals (3). Furthermore, the activation of KYNA production was not restrained in resident immune cells of adipose tissue as the increased expression of IDO1, KYAT2 and KYAT3 can also be found in adipocytes. 
This up-regulation may be due to the increased production of proinflammatory cytokines from resident immune cells since adipocyte does not express KMO (3). However, in another study, Pyun et al. found a negative correlation between serum KYNA levels and BMI (6). These controversial observations may be due to the different criteria. Another possible explanation to the controversy is that the KYNA determination method used in these studies are different: the last one used an ELISA kit to determinate serum KYNA levels, while the others used the HPLC-MS/MS method. It needs to be further verified whether these divergent results are due to some immeasurable confounding factors.

Liver seems to be another important source of serum KYNA in overweight individuals as TDO and KYATs are highly expressed in this organ $(17,18)$. Moreover, overweight is frequently associated with a low-grade chronic inflammation with an induction of IDO1 in liver (62). Furthermore, both of the liver and adipose tissue have a closed crosstalk between resident immune cells (Kupffer cells or macrophages) and metabolic cells (adipocytes or hepatocytes). However, to the best of our knowledge, neither the production of KYNA nor the regulation of IDO, TDO and KYATs in hepatocytes under metabolic challenge have been reported.

\section{Physical Exercise, the Second Link Between KYNA and Metabolic Diseases}

Physical exercise has been described as a promising nonpharmacological treatment for overweight and some other metabolic diseases (63). In addition to its role in energy expenditure regulation, skeletal muscle is increasingly considered as one of the largest endocrine organs in our body. It secretes a variety of myokines and bioactive metabolites, which exerts important effects on the regulation of metabolism and inflammation. KYNA can also be synthesized by skeletal muscle and its production is closely correlated to the physical exercise in both human and mouse $(11,64)$.

All the four KYATs are expressed in skeletal muscle, but KYAT isoforms display fiber-type specific expression. KYAT1, KYAT3 and KYAT4 are more abundant in oxidative type I than glycolytic type II fiber (65). Accordingly, an increased serum KYNA level has been found in individuals after endurance exercise (64). Within the first hour after aerobic exercise, there is an increase in plasmatic KYNA and this effect lasts for 2 hours after exercise. In contrast, high-intensity eccentric exercise did not lead to increased plasmatic KYNA concentration (64). Regarding the effect of long-term exercise on KYNA, a recent study of 4-week physical exercises on human found that physical exercises promote an increase in the amount of KYNA in sweat on day 14. The KYNA level returned to baseline on day 28 (66). Additionally, inhibition of KYATs reduces myotube oxidative capacity and exercise performance in mice (67).

Physical exercise induces peroxisome-proliferator activated receptor $\gamma$ coactivator $1 \alpha$ (PGC- $1 \alpha$ ) expression in skeletal muscle (11). PGC1-1 $\alpha$ coordinates the expression of several genes involved in the adaptive energy metabolism and fatigue-resistance such as mitochondrial biogenesis and fatty acid oxidation. Recently, the canonical and longest transcript variant of PGC- $1 \alpha$, PGC- $1 \alpha 1$, is reported to up-regulate KYAT2 and KYAT4 expression (11). Such mechanism in skeletal muscle during physical exercise may be primarily aimed at enhancing the malate-aspartate shuttle as both KYATs are important enzymes in the malate-aspartate shuttle (67). Consequently, this mechanism shifts the kynurenine metabolism to KYNA production.

This exercise-induced KYNA production is originally described as the crosstalk between skeletal muscle and the brain to elucidate the effectiveness of exercise in reducing depressive symptoms. A recent study by Agudelo et al. demonstrated that KYNA increases energy utilization by activating G-protein-coupled receptor 35 (GPR35), which stimulates lipid metabolism, thermogenic, and anti-inflammatory gene expression in adipose tissue (68). Also, GPR35 agonists was reported to suppress high fat diet-induced fatty liver development (5). These data uncovered that skeletal muscle derived from KYNA may be a potential regulator of energy homeostasis and a coordinator of exercise-induced adaptations in other organs including liver, adipose tissue and brain.

However, it should be noted that physical exercise induces strong and transit increases in KYNA levels while inflammation leads to mild and sustained increases in KYNA levels.

\section{Perinatal Nutrition, The Third Link Between KYNA and Metabolic Diseases}

Epidemiological and experimental data have suggested that perinatal nutrition has a significant role in the development of lifelong metabolic disorders (69). KYNA may also act as a link between perinatal offspring and mother. KYNA can pass through the placenta into the fetus (70). However, placental and fetal KYNA were not affected by placental infusion of L-KYN in mice (70). Also, under physiological conditions, KYNA was higher in the liver and brain of mouse's fetuses than in the placenta, and KYNA in the fetus was not affected during oral maternal administration of KYNA. It can be hypothesized that maternal KYNA cannot affect fetus through placenta $(70,71)$.

Although maternal KYNA cannot directly affect the fetus, some studies found the KYNA content in breast milk gradually increases in different lactation periods (10). Epidemiological studies showed a slower body weight gain in naturally fed newborns compared to artificially fed ones (72). Although the formula milk powder for infants in different periods are different, studies have found that KYNA content in formula milk powder is much lower than that in breast milk (10). Rats postnatally exposed to KYNA supplementation were observed to have a significant reduction of body weight gain, but no changes in total body surface and bone mineral density. The rat offspring supplemented with KYNA presents a lower mass gain during the first 21 days of life, which indicates that KYNA may act as an anti-obese agent (10).

Another potential mechanism is that perinatal KYNA may be protective against overweight by modulating the gut microbiota. Formula feeding appears to promote the microbiota associated with overweight (73), while KYNA stimulates the growth of certain probiotics (74). It still needs to be further explored whether the presence of KYNA in breast milk acts as a modulator of gut microbiota. 


\section{POTENTIAL MECHANISM OF KYNA INVOLVED IN COMMON METABOLIC DISEASES}

\section{Glutamate Receptors}

KYNA is well-known for its role as an endogenous N-methyl-Daspartate receptor (NMDAR) antagonist in the brain. In the periphery, the expression of functional NMDA receptor is reported in the pancreatic $\beta$-cell. Activation of NMDA receptor reduces the glucose-stimulated insulin secretion. Likewise, NMDA receptor knockout in mouse islets increases glucose-stimulated insulin secretion. NMDA activation in $\beta$-cells also promotes cell death under stress. In microphages, activation of NMDA receptor induces ABCA1 degradation which promotes cholesterol accumulation and foam cell formation (75). In liver, NMDA receptor is present on the surface of Kupffer cells, and its activation has been reported to limit inflammasome activation (76).

KYNA acts as a low affinity competitive antagonist of AMPA $(\alpha-$ amino-3-hydroxy-5-methyl-4-isoxazole propionic acid) receptors. It directly acts on the glutamate binding domain. Meanwhile, low concentrations $(0.03-30 \mu \mathrm{M})$ of KYNA potentiate AMPA receptor responses (77). Therefore, KYNA has a dual action on AMPA receptor responses.

KYNA also directly interacts with the glutamate-binding domain of kainate receptors. Its antagonistic effect on this type of receptor is the least potent (IC50 $500 \mu \mathrm{M}$ ) among the 3 types of glutamate receptors (78).

However, serum KYNA level hardly reaches to micromolar levels. It is unclear whether KYNA in serum or in the periphery is sufficient to antagonize these glutamate receptors.

\section{Other High Affinity Receptors}

The G-protein-coupled receptor 35 (GPR35) is an orphan receptor that was identified in 1998 (79). It was originally described as a receptor for zaprinast, a phosphodiesterase (PDE) inhibitor. Recently, the KYNA was identified as an endogenous ligand for GPR35 with an $\mathrm{EC}_{50}$ of $39 \mu \mathrm{M}$ in human and $7.9 \mu \mathrm{M}$ in rat (80). Although the plasmatic concentration of KYNA is often in the nanomolar range in humans, it can become micromolar under inflammatory conditions.

GPR35 is associated with Gi/G0 and G13 proteins (81). Thus, activation of GPR35 reduces the activity of adenylate cyclase $(\mathrm{Gi} /$ G0) and/or increases that of the RhoA pathway (G13). GPR35 is expressed in central nervous system and in many peripheral tissues. In humans, significant expression of GPR35 has been detected in the colon, pancreas, small intestine, spleen and immune cells (monocytes, neutrophils, $\mathrm{T}$ cells and dendritic cells). The level of its expression is lower in the stomach, skeletal muscle, adipose tissue, pancreatic islets, kidney, liver, and thymus (82). Activation of GPR35 by KYNA has anti-inflammatory effect (83) by inducing autophagy-dependent degradation of NLRP3 in macrophage (84). It also plays anti-nociceptive (85) and anti-asthmatic (86) roles. Furthermore, KYNA enhances Pgc- $1 \alpha 1$ and UCP1 expression GPR35 signaling in adipocytes, which suggests KYNA is a signaling molecule which directly controls energy homeostasis (68).
More recently, KYNA has been discovered to significantly increase AMP-activated protein kinase (AMPK) phosphorylation and to ameliorate palmitate-induced inflammation and insulin resistance. It potentially alleviates inflammation and insulin resistance in skeletal muscle and adipose tissues through GPR35/ AMPK and SIRT6-mediated pathways (87). It may also ameliorate hepatic steatosis via the AMPK/autophagy- and AMPK/ORP150mediated suppression of endoplasmic reticulum stress (6).

KYNA is also identified as an endogenous ligand for Aryl hydrocarbon receptor (AhR). AhR was originally described as a xenobiotic receptor, also known as the dioxin receptor. It is activated by exogenous ligands, such as flavonoids, natural plant polyphenols, indoles and dioxins. AhR plays multiple roles in xenobiotic metabolism, the regulation of inflammation, development, and the homeostasis of several organs (88). DiNatale and colleagues (89) showed that KYNA is a potent endogenous agonist of AHR with an $\mathrm{EC}_{25}$ around 100nM. Activation of AHR by KYNA may lead to IL6 expression in tumor cells.

Moreover, studies have shown that KYNA can also act at nicotinic receptors as a potent noncompetitive antagonist, particularly at the $\alpha 7$ subunit of the nicotinic receptor (90). KYNA inhibits CHRNA7 in a non-competitive manner at physiological concentrations. CHRNA7 was found to be expressed in glutamatergic axon terminals. Activation of CHRNA7 enhances glutamate release. Thus, KYNA may also be involved in the repression of glutamate release at the presynaptic level. This represents another mechanism by which KYNA exerts its anti-glutamatergic effect (90). The characteristics of KYNA receptors are summarized in Table 2.

\section{Scavenger of Free Radicals}

In addition to its receptor-dependent effects, KYNA at high concentrations $(100-300 \mu \mathrm{M})$ also acts as a potent endogenous antioxidant, as it is a scavenger of free radicals such as hydroxyl radicals $(\mathrm{OH})$, superoxide anion $\left(\mathrm{O}_{2}^{-}\right)$and peroxynitrite $\left(\mathrm{ONOO}^{-}\right)$ (92). Since oxidative stress is also critical for the pathogenesis of metabolic diseases (93), the antioxidative properties of KYNA represent an important mechanism in preventing the onset of metabolic diseases.

Interestingly, another study showed that KYNA is not a guaranteed protector against oxidative stress. It exhibits a strong pro-oxidative effect combined with $\delta$-aminolaevulinic acid (ALA), an endogenous precursor of heme and source of hydroxyl radical, and elevates deoxyribose deterioration by 9 times compared to ALA alone (94).

\section{Mitochondrial Homeostasis}

The mitochondrial localization of KYAT suggests a direct release of KYNA into the mitochondria. KYNA plays a key role in the

TABLE 2 | The characteristic of KYNA receptor.

\begin{tabular}{llcc}
\hline Receptor & KYNA & Affinity & References \\
\hline NMDA & antagonist & $\mathrm{IC}_{50} 7.9-20 \mu \mathrm{M}$ & $(70,91)$ \\
Kainate & antagonist & $\mathrm{IC}_{50} 500 \mu \mathrm{M}$ & $(78)$ \\
CHRNA7 & antagonist & $\mathrm{IC}_{50} 7 \mu \mathrm{M}$ & $(90)$ \\
GPR35 & agonist & $\mathrm{EC}_{50} 8-40 \mu \mathrm{M}$ & $(80)$ \\
AHR & agonist & $\mathrm{EC}_{25} 100 \mathrm{nM}$ & $(89)$
\end{tabular}


TABLE 3 | The roles of KYNA in metabolic diseases.

\begin{tabular}{|c|c|c|c|}
\hline Organ/cell & KYNA production & Effects & $\begin{array}{l}\text { Associated } \\
\text { metabolic } \\
\text { disease }\end{array}$ \\
\hline Liver & $\begin{array}{l}\text { High expression levels of TDO/IDO/KYATs were detected } \\
\text { in liver (12) }\end{array}$ & $\begin{array}{l}\text { Activation of GPR35 inhibits the development of NAFLD (5) } \\
\text { Inhibition of Kuffer cells NMDA receptor by KYNA limits inflammasome } \\
\text { activation (76) } \\
\text { Activation of AMPK/autophagy- and AMPK/ORP150 pathway by KYNA } \\
\text { ameliorate endoplasmic reticulum stress and hepatic steatosis (6) }\end{array}$ & $\begin{array}{l}\text { NAFLD } \\
\text { Metabolic } \\
\text { inflammation } \\
\text { Hepatic steatosis }\end{array}$ \\
\hline $\begin{array}{l}\text { Adipose } \\
\text { tissue/ } \\
\text { adipocyte }\end{array}$ & $\begin{array}{l}\text { Expression of IDO1/KYAT1/KYAT3 were detected in } \\
\text { adipocytes (3) }\end{array}$ & $\begin{array}{l}\text { Activation of GPR35 by KYNA promotes the expression of PGC1- } \alpha \text { and } \\
\text { UCP1 (67) } \\
\text { Activation of GPR35/AMPK and SIRT6 pathways by KYNA reduces } \\
\text { inflammation and insulin resistance in adipocytes ( } 87 \text { ) }\end{array}$ & $\begin{array}{l}\text { Insulin resistance } \\
\text { Metabolic } \\
\text { inflammation }\end{array}$ \\
\hline Muscle & $\begin{array}{l}\text { Endurance essences enhance KYATs expression and } \\
\text { promote KYNA production }(64,65,67)\end{array}$ & $\begin{array}{l}\text { Activation of GPR35/AMPK and SIRT6 pathways by KYNA reduces } \\
\text { inflammation and insulin resistance in skeletal muscle ( } 87 \text { ) }\end{array}$ & $\begin{array}{l}\text { Insulin resistance } \\
\text { Metabolic } \\
\text { inflammation }\end{array}$ \\
\hline Immune cell & $\begin{array}{l}\text { KYAT1/KYAT2 expressions were detected in in both } \\
\text { unstimulated and stimulated macrophage (107) }\end{array}$ & $\begin{array}{l}\text { Antagonize NMDA receptor by KYNA inhibits ABCA1 degradation (75) } \\
\text { Activation of GPR35 by KYNA induces autophagy-dependent } \\
\text { degradation of NLRP3 in macrophage (84) }\end{array}$ & $\begin{array}{l}\text { Cholesterol } \\
\text { accumulation } \\
\text { Metabolic } \\
\text { inflammation }\end{array}$ \\
\hline Pancreas & $\begin{array}{l}\text { KYNA were detected in pancreas fluid, expression of } \\
\text { TDO/KYATs were detected in pancreatic islets }(9,13)\end{array}$ & $\begin{array}{l}\text { High concentration of KYNA enhances glucose stimulated insulin } \\
\text { secretion (13) }\end{array}$ & Type 2 diabetes \\
\hline $\begin{array}{l}\text { Mammary } \\
\text { gland }\end{array}$ & $\begin{array}{l}\text { KYNA content in breast milk gradually increases in } \\
\text { different lactation periods (10) }\end{array}$ & KYNA may act as an anti-obese agent for children (10) & Obesity \\
\hline
\end{tabular}

redox balance in the mitochondria. The expression and function of KYATs has been shown to be diminished in rat model with mitochondrial dysfunction $(95,96)$.

Firstly, the formation of KYNA diverts the pathway from de novo synthesis of $\mathrm{NAD}+\mathrm{NADH}$, which regulates the mitochondrial TCA cycle, oxidative state and mitochondrial dynamics, suggesting the involvement of KYNA in the mitochondria energy metabolism regulation. MPTP and 3-nitropropionic acid (3-NA) have inhibitory effects on mitochondrial respiratory chain complexes and on KYAT1 and KYAT2, thus compromise the ATP and KYNA production in the mitochondria (96). Experimentally, FK506, a neuroimmunophilin drug, not only enhanced the formation of KYNA, but abolished the inhibition of KYNA synthesis caused by MPTP and 3-NA. This result suggested that the restoration of respiratory chain function may activate the KYNA synthesis pathway (97). In the case of monogenic form of Leigh Syndrome, the loss-of-function mutation in LRPPRC causes mitochondrial RNA metabolism disorder. The metabolic signature demonstrated a decrease in kynurenine, the precursor of KYNA (98). In patients with Schizophrenia, the prescription of $\mathrm{N}$-acetylcysteine has shown inhibitory effect on KYAT, decreasing the deleterious effect of elevated KYNA on glutamate and dopamine signaling (99). Furthermore, the plasmatic KYNA is positively correlated with fatty acid oxidation and mitochondrial proliferation in the liver of rat (100).

Secondly, KYNA has shown scavenging property of $\mathrm{OH} \boldsymbol{}, \mathrm{O}_{2}{ }^{-}$, $\mathrm{ONOO}^{-}(92,101)$. In a preparation of oocytes, KYNA significantly reduced ROS and lipid peroxidation induced by $\mathrm{FeSO}_{4}$. For 3Methylglutaric acid (3MGA) which accumulates in the brains of children coursing with metabolic acidurias, experiments showed that 3MGA induced an increase in ROS production and lipid peroxidation and a decrease in mitochondrial function. Addition of KYNA showed antagonist effects (102).

Thirdly, as has been discussed in 4.1, KYNA has potent antagonist effects over NMDAR (103), which may reduce de notorious effect of excitotoxicity on mitochondria, via decreasing excessive intracellular $\mathrm{Ca}^{2+}$ as example (104).

Finally, KYNA was shown to directly impair respiratory parameters of heart mitochondria. Moreover, the effect is selective for complex I $(105,106)$. However, this respiratory chainmodulating property was only observed in heart mitochondria, and is absent for brain and liver mitochondria, suggesting profound differences between tissular mitochondria content and helping to explain the tissue-specific effect of KYNA. It should be pointed out that these in vivo experiments were carried out with high concentration of KYNA $(125-1000 \mu \mathrm{M})$ which is rarely achieved under physiological conditions (92).

\section{CONCLUSION}

Increasing evidence indicates that KYNA can act as a signaling molecule to regulate energy expenditure in a network integrating nutrition, physical exercise, inflammation and metabolic diseases besides its neuro-protector role in the central nervous system (Table 3). Targeting KYNA signaling network or its metabolic pathway harbors high potentials to expand the range of strategy to prevent and treat metabolic diseases.

\section{AUTHOR CONTRIBUTIONS}

ZS designed and reviewed the article. DZ wrote the draft. XDZ revised the content. JL collected references. All authors contributed to the article and approved the submitted version.

\section{FUNDING}

This research was funded by the Henan Provincial Postdoctorate Research Fund and ENN Research Fund. 


\section{REFERENCES}

1. Turski MP, Turska M, Paluszkiewicz P, Parada-Turska J, Oxenkrug GF. Kynurenic Acid in the Digestive System-New Facts, New Challenges. Int $J$ Tryptophan Res (2013) 6:47-55. doi: 10.4137/IJTR.S12536

2. Wirthgen E, Hoeflich A, Rebl A, Gunther J. Kynurenic Acid: The JanusFaced Role of an Immunomodulatory Tryptophan Metabolite and Its Link to Pathological Conditions. Front Immunol (2017) 8:1957. doi: 10.3389/ fimmu.2017.01957

3. Favennec M, Hennart B, Caiazzo R, Leloire A, Yengo L, Verbanck M, et al. The Kynurenine Pathway Is Activated in Human Obesity and Shifted Toward Kynurenine Monooxygenase Activation. Obes (Silver Spring) (2015) 23(10):2066-74. doi: 10.1002/oby.21199

4. Oxenkrug GF. Increased Plasma Levels of Xanthurenic and Kynurenic Acids in Type 2 Diabetes. Mol Neurobiol (2015) 52(2):805-10. doi: 10.1007/ s12035-015-9232-0

5. Nam SY, Park SJ, Im DS. Protective Effect of Lodoxamide on Hepatic Steatosis Through GPR35. Cell Signal (2019) 53:190-200. doi: 10.1016/ j.cellsig.2018.10.001

6. Pyun DH, Kim TJ, Kim MJ, Hong SA, Abd El-Aty AM, Jeong JH, et al. Endogenous Metabolite, Kynurenic Acid, Attenuates Nonalcoholic Fatty Liver Disease via AMPK/autophagy- and AMPK/ORP150-Mediated Signaling. J Cell Physiol (2021) 236(7):4902-12. doi: 10.1002/jcp.30199

7. Cogo A, Mangin G, Maier B, Callebert J, Mazighi M, Chabriat H, et al. Increased Serum QUIN/KYNA Is a Reliable Biomarker of Post-Stroke Cognitive Decline. Mol Neurodegener (2021) 16(1):7. doi: 10.1186/s13024020-00421-4

8. Kuc D, Rahnama M, Tomaszewski T, Rzeski W, Wejksza K, UrbanikSypniewska T, et al. Kynurenic Acid in Human Saliva-Does it Influence Oral Microflora? Pharmacol Rep (2006) 58(3):393-8.

9. Paluszkiewicz P, Zgrajka W, Saran T, Schabowski J, Piedra JL, Fedkiv O, et al. High Concentration of Kynurenic Acid in Bile and Pancreatic Juice. Amino Acids (2009) 37(4):637-41. doi: 10.1007/s00726-008-0183-x

10. Milart P, Paluszkiewicz P, Dobrowolski P, Tomaszewska E, Smolinska K, Debinska I, et al. Kynurenic Acid as the Neglected Ingredient of Commercial Baby Formulas. Sci Rep (2019) 9(1):6108. doi: 10.1038/ s41598-019-42646-4

11. Agudelo LZ, Femenia T, Orhan F, Porsmyr-Palmertz M, Goiny M, Martinez-Redondo V, et al. Skeletal Muscle PGC-1alphal Modulates Kynurenine Metabolism and Mediates Resilience to Stress-Induced Depression. Cell (2014) 159(1):33-45. doi: 10.1016/j.cell.2014.07.051

12. Pawlak D, Tankiewicz A, Matys T, Buczko W. Peripheral Distribution of Kynurenine Metabolites and Activity of Kynurenine Pathway Enzymes in Renal Failure. J Physiol Pharmacol (2003) 54(2):175-89.

13. Liu JJ, Raynal S, Bailbe D, Gausseres B, Carbonne C, Autier V, et al. Expression of the Kynurenine Pathway Enzymes in the Pancreatic Islet Cells. Activation by Cytokines and Glucolipotoxicity. Biochim Biophys Acta (2015) 1852(5):980-91. doi: 10.1016/j.bbadis.2015.02.001

14. Stazka J, Luchowski P, Wielosz M, Kleinrok Z, Urbanska EM. EndotheliumDependent Production and Liberation of Kynurenic Acid by Rat Aortic Rings Exposed to L-Kynurenine. Eur J Pharmacol (2002) 448(2-3):133-7. doi: 10.1016/S0014-2999(02)01943-X

15. Jones SP, Franco NF, Varney B, Sundaram G, Brown DA, de Bie J, et al. Expression of the Kynurenine Pathway in Human Peripheral Blood Mononuclear Cells: Implications for Inflammatory and Neurodegenerative Disease. PLoS One (2015) 10(6):e0131389. doi: 10.1371/journal.pone.0131389

16. Hiratsuka C, Fukuwatari T, Shibata K. Fate of Dietary Tryptophan in Young Japanese Women. Int J Tryptophan Res (2012) 5:33-47. doi: 10.4137/ IJTR.S10497

17. Batabyal D, Yeh SR. Human Tryptophan Dioxygenase: A Comparison to Indoleamine 2,3-Dioxygenase. J Am Chem Soc (2007) 129(50):15690-701. doi: $10.1021 / \mathrm{ja} 076186 \mathrm{k}$

18. Consortium GT. The Genotype-Tissue Expression (GTEx) Project. Nat Genet (2013) 45(6):580-5. doi: 10.1038/ng.2653

19. Theate I, van Baren N, Pilotte L, Moulin P, Larrieu P, Renauld JC, et al. Extensive Profiling of the Expression of the Indoleamine 2,3-Dioxygenase 1 Protein in Normal and Tumoral Human Tissues. Cancer Immunol Res (2015) 3(2):161-72. doi: 10.1158/2326-6066.CIR-14-0137
20. van Baren N, Van den Eynde BJ. Tryptophan-Degrading Enzymes in Tumoral Immune Resistance. Front Immunol (2015) 6:34. doi: 10.3389/ fimmu.2015.00034

21. Han Q, Li J, Li J.pH Dependence, Substrate Specificity and Inhibition of Human Kynurenine Aminotransferase I. Eur J Biochem (2004) 271(2324):4804-14. doi: 10.1111/j.1432-1033.2004.04446.x

22. Cooper AJ, Pinto JT. Cysteine S-Conjugate Beta-Lyases. Amino Acids (2006) 30(1):1-15. doi: 10.1007/s00726-005-0243-4

23. Okuno E, Nishikawa T, Nakamura M. Kynurenine Aminotransferases in the Rat. Localization and Characterization. Adv Exp Med Biol (1996) 398:45564. doi: 10.1007/978-1-4613-0381-7_71

24. Mosca M, Croci C, Mostardini M, Breton J, Malyszko J, Avanzi N, et al. Tissue Expression and Translational Control of Rat Kynurenine Aminotransferase/Glutamine Transaminase K mRNAs. Biochim Biophys Acta (2003) 1628(1):1-10. doi: 10.1016/S0167-4781(03)00071-X

25. Kapoor R, Lim KS, Cheng A, Garrick T, Kapoor V. Preliminary Evidence for a Link Between Schizophrenia and NMDA-Glycine Site Receptor Ligand Metabolic Enzymes, D-Amino Acid Oxidase (DAAO) and Kynurenine Aminotransferase-1 (KAT-1). Brain Res (2006) 1106(1):205-10. doi: 10.1016/j.brainres.2006.05.082

26. Kwok JB, Kapoor R, Gotoda T, Iwamoto Y, Iizuka Y, Yamada N, et al. A Missense Mutation in Kynurenine Aminotransferase-1 in Spontaneously Hypertensive Rats. J Biol Chem (2002) 277(39):35779-82. doi: 10.1074/ jbc.C200303200

27. Guillemin GJ, Kerr SJ, Smythe GA, Smith DG, Kapoor V, Armati PJ, et al. Kynurenine Pathway Metabolism in Human Astrocytes: A Paradox for Neuronal Protection. J Neurochem (2001) 78(4):842-53. doi: 10.1046/j.14714159.2001.00498.x

28. Han Q, Cai T, Tagle DA, Robinson H, Li J. Substrate Specificity and Structure of Human Aminoadipate Aminotransferase/Kynurenine Aminotransferase II. Biosci Rep (2008) 28(4):205-15. doi: 10.1042/ BSR20080085

29. Guidetti P, Amori L, Sapko MT, Okuno E, Schwarcz R. Mitochondrial Aspartate Aminotransferase: A Third Kynurenate-Producing Enzyme in the Mammalian Brain. J Neurochem (2007) 102(1):103-11. doi: 10.1111/j.14714159.2007.04556.x

30. Guidetti P, Hoffman GE, Melendez-Ferro M, Albuquerque EX, Schwarcz R. Astrocytic Localization of Kynurenine Aminotransferase II in the Rat Brain Visualized by Immunocytochemistry. Glia (2007) 55(1):78-92. doi: 10.1002/ glia.20432

31. Han Q, Robinson H, Cai T, Tagle DA, Li J. Biochemical and Structural Properties of Mouse Kynurenine Aminotransferase III. Mol Cell Biol (2009) 29(3):784-93. doi: 10.1128/MCB.01272-08

32. Yang C, Zhang L, Han Q, Liao C, Lan J, Ding H, et al. Kynurenine Aminotransferase 3/Glutamine Transaminase L/cysteine Conjugate BetaLyase 2 Is a Major Glutamine Transaminase in the Mouse Kidney. Biochem Biophys Rep (2016) 8:234-41. doi: 10.1016/j.bbrep.2016.09.008

33. Borst P. The Malate-Aspartate Shuttle (Borst Cycle): How it Started and Developed Into a Major Metabolic Pathway. IUBMB Life (2020) 72 (11):2241-59. doi: 10.1002/iub.2367

34. Robinson BH, Halperin ML. Transport of Reduced Nicotinamide-Adenine Dinucleotide Into Mitochondria of Rat White Adipose Tissue. Biochem J (1970) 116(2):229-33. doi: 10.1042/bj1160229

35. Hirai K, Kuroyanagi H, Tatebayashi Y, Hayashi Y, Hirabayashi-Takahashi K, Saito K, et al. Dual Role of the Carboxyl-Terminal Region of Pig Liver LKynurenine 3-Monooxygenase: Mitochondrial-Targeting Signal and Enzymatic Activity. J Biochem (2010) 148(6):639-50. doi: 10.1093/jb/mvq099

36. Castellano-Gonzalez G, Jacobs KR, Don E, Cole NJ, Adams S, Lim CK, et al. Kynurenine 3-Monooxygenase Activity in Human Primary Neurons and Effect on Cellular Bioenergetics Identifies New Neurotoxic Mechanisms. Neurotox Res (2019) 35(3):530-41. doi: 10.1007/s12640-019-9997-4

37. Comai S, Costa CV, Ragazzi E, Bertazzo A, Allegri G. The Effect of Age on the Enzyme Activities of Tryptophan Metabolism Along the Kynurenine Pathway in Rats. Clin Chim Acta (2005) 360(1-2):67-80. doi: 10.1016/ j.cccn.2005.04.013

38. Alberati-Giani D, Cesura AM, Broger C, Warren WD, Rover S, Malherbe P. Cloning and Functional Expression of Human Kynurenine 3-Monooxygenase. FEBS Lett (1997) 410(2-3):407-12. doi: 10.1016/S0014-5793(97)00627-3 
39. Lowe DM, Gee M, Haslam C, Leavens B, Christodoulou E, Hissey P, et al. Lead Discovery for Human Kynurenine 3-Monooxygenase by HighThroughput RapidFire Mass Spectrometry. J Biomol Screen (2014) 19 (4):508-15. doi: 10.1177/1087057113518069

40. Winkler D, Beconi M, Toledo-Sherman LM, Prime M, Ebneth A, Dominguez C, et al. Development of LC/MS/MS, High-Throughput Enzymatic and Cellular Assays for the Characterization of Compounds That Inhibit Kynurenine Monooxygenase (KMO). J Biomol Screen (2013) 18 (8):879-89. doi: 10.1177/1087057113489731

41. Phillips RS, Iradukunda EC, Hughes T, Bowen JP. Modulation of Enzyme Activity in the Kynurenine Pathway by Kynurenine Monooxygenase Inhibition. Front Mol Biosci (2019) 6:3. doi: 10.3389/fmolb.2019.00003

42. Kubo H, Hoshi M, Mouri A, Tashita C, Yamamoto Y, Nabeshima T, et al. Absence of Kynurenine 3-Monooxygenase Reduces Mortality of Acute Viral Myocarditis in Mice. Immunol Lett (2017) 181:94-100. doi: 10.1016/ j.imlet.2016.11.012

43. Cote F, Thevenot E, Fligny C, Fromes Y, Darmon M, Ripoche MA, et al. Disruption of the Nonneuronal Tph1 Gene Demonstrates the Importance of Peripheral Serotonin in Cardiac Function. Proc Natl Acad Sci USA (2003) 100(23):13525-30. doi: 10.1073/pnas.2233056100

44. Cichon S, Winge I, Mattheisen M, Georgi A, Karpushova A, Freudenberg J, et al. Brain-Specific Tryptophan Hydroxylase 2 (TPH2): A Functional Pro206Ser Substitution and Variation in the 5 -Region Are Associated With Bipolar Affective Disorder. Hum Mol Genet (2008) 17(1):87-97. doi: $10.1093 / \mathrm{hmg} / \mathrm{ddm} 286$

45. Littlejohn TK, Takikawa O, Truscott RJ, Walker MJ. Asp274 and His346 are Essential for Heme Binding and Catalytic Function of Human Indoleamine 2,3-Dioxygenase. J Biol Chem (2003) 278(32):29525-31. doi: 10.1074/ jbc.M301700200

46. Lima S, Khristoforov R, Momany C, Phillips RS. Crystal Structure of Homo Sapiens Kynureninase. Biochemistry (2007) 46(10):2735-44. doi: 10.1021/ bi0616697

47. Ramos-Chavez LA, Lugo Huitron R, Gonzalez Esquivel D, Pineda B, Rios C, Silva-Adaya D, et al. Relevance of Alternative Routes of Kynurenic Acid Production in the Brain. Oxid Med Cell Longev (2018) 2018:5272741. doi: $10.1155 / 2018 / 5272741$

48. Bahn A, Ljubojevic M, Lorenz H, Schultz C, Ghebremedhin E, Ugele B, et al. Murine Renal Organic Anion Transporters Moat1 and Moat3 Facilitate the Transport of Neuroactive Tryptophan Metabolites. Am J Physiol Cell Physiol (2005) 289(5):C1075-84. doi: 10.1152/ajpcell.00619.2004

49. Granados JC, Richelle A, Gutierrez JM, Zhang P, Zhang X, Bhatnagar V, et al. Coordinate Regulation of Systemic and Kidney Tryptophan Metabolism by the Drug Transporters OAT1 and OAT3. J Biol Chem (2021) 296:100575. doi: 10.1016/j.jbc.2021.100575

50. Nemeth H, Toldi J, Vecsei L. Role of Kynurenines in the Central and Peripheral Nervous Systems. Curr Neurovasc Res (2005) 2(3):249-60. doi: $10.2174 / 1567202054368326$

51. Swartz KJ, During MJ, Freese A, Beal MF. Cerebral Synthesis and Release of Kynurenic Acid: An Endogenous Antagonist of Excitatory Amino Acid Receptors. J Neurosci (1990) 10(9):2965-73. doi: 10.1523/JNEUROSCI.1009-02965.1990

52. Lin L, Lemieux GA, Enogieru OJ, Giacomini KM, Ashrafi K. Neural Production of Kynurenic Acid in Caenorhabditis Elegans Requires the AAT-1 Transporter. Genes Dev (2020) 34(15-16):1033-8. doi: 10.1101/ gad. 339119.120

53. Sekine A, Okamoto M, Kanatani Y, Sano M, Shibata K, Fukuwatari T. Amino Acids Inhibit Kynurenic Acid Formation via Suppression of Kynurenine Uptake or Kynurenic Acid Synthesis in Rat Brain In Vitro. Springerplus (2015) 4:48. doi: 10.1186/s40064-015-0826-9

54. Sinclair LV, Neyens D, Ramsay G, Taylor PM, Cantrell DA. Single Cell Analysis of Kynurenine and System L Amino Acid Transport in T Cells. Nat Commun (2018) 9(1):1981. doi: 10.1038/s41467-018-04366-7

55. Mandi Y, Endresz V, Mosolygo T, Burian K, Lantos I, Fulop F, et al. The Opposite Effects of Kynurenic Acid and Different Kynurenic Acid Analogs on Tumor Necrosis Factor-Alpha (TNF-Alpha) Production and Tumor Necrosis Factor-Stimulated Gene-6 (TSG-6) Expression. Front Immunol (2019) 10:1406. doi: 10.3389/fimmu.2019.01406
56. Malaczewska J, Siwicki AK, Wojcik RM, Kaczorek E, Turski WA. Effect of Oral Administration of Kynurenic Acid on the Activity of the Peripheral Blood Leukocytes in Mice. Cent Eur J Immunol (2014) 39(1):6-13. doi: 10.5114/ceji.2014.42115

57. Hotamisligil GS. Inflammation and Metabolic Disorders. Nature (2006) 444 (7121):860-7. doi: 10.1038/nature05485

58. Saltiel AR, Olefsky JM. Inflammatory Mechanisms Linking Obesity and Metabolic Disease. J Clin Invest (2017) 127(1):1-4. doi: 10.1172/JCI92035

59. Oxenkrug G, Cornicelli J, van der Hart M, Roeser J, Summergrad P. Kynurenic Acid, an Aryl Hydrocarbon Receptor Ligand, Is Elevated in Serum of Zucker Fatty Rats. Integr Mol Med (2016) 3(4):761-3.

60. Lauterbach MA, Latz E, Christ A. Metabolomic Profiling Reveals Distinct and Mutual Effects of Diet and Inflammation in Shaping Systemic Metabolism in Ldlr(-/-) Mice. Metabolites (2020) 10(9). doi: 10.3390/ metabo 10090336

61. Ho JE, Larson MG, Ghorbani A, Cheng S, Chen MH, Keyes M, et al. Metabolomic Profiles of Body Mass Index in the Framingham Heart Study Reveal Distinct Cardiometabolic Phenotypes. PLoS One (2016) 11(2): e0148361. doi: 10.1371/journal.pone.0148361

62. Chaves Filho AJM, Lima CNC, Vasconcelos SMM, de Lucena DF, Maes M, Macedo D. IDO Chronic Immune Activation and Tryptophan Metabolic Pathway: A Potential Pathophysiological Link Between Depression and Obesity. Prog Neuropsychopharmacol Biol Psychiatry (2018) 80(Pt C):23449. doi: 10.1016/j.pnpbp.2017.04.035

63. Yang Z, Scott CA, Mao C, Tang J, Farmer AJ. Resistance Exercise Versus Aerobic Exercise for Type 2 Diabetes: A Systematic Review and MetaAnalysis. Sports Med (2014) 44(4):487-99. doi: 10.1007/s40279-013-0128-8

64. Schlittler M, Goiny M, Agudelo LZ, Venckunas T, Brazaitis M, Skurvydas A, et al. Endurance Exercise Increases Skeletal Muscle Kynurenine Aminotransferases and Plasma Kynurenic Acid in Humans. Am J Physiol Cell Physiol (2016) 310(10):C836-40. doi: 10.1152/ajpcell.00053.2016

65. Wyckelsma VL, Lindkvist W, Venckunas T, Brazaitis M, Kamandulis S, Paasuke M, et al. Kynurenine Aminotransferase Isoforms Display FiberType Specific Expression in Young and Old Human Skeletal Muscle. Exp Gerontol (2020) 134:110880. doi: 10.1016/j.exger.2020.110880

66. Saran T, Turska M, Kocki T, Zawadka M, Zielinski G, Turski WA, et al. Effect of 4-Week Physical Exercises on Tryptophan, Kynurenine and Kynurenic Acid Content in Human Sweat. Sci Rep (2021) 11(1):11092. doi: 10.1038/s41598-021-90616-6

67. Agudelo LZ, Ferreira DMS, Dadvar S, Cervenka I, Ketscher L, Izadi M, et al. Skeletal Muscle PGC-1alpha1 Reroutes Kynurenine Metabolism to Increase Energy Efficiency and Fatigue-Resistance. Nat Commun (2019) 10(1):2767. doi: $10.1038 /$ s41467-019-10712-0

68. Agudelo LZ, Ferreira DMS, Cervenka I, Bryzgalova G, Dadvar S, Jannig PR, et al. Kynurenic Acid and Gpr35 Regulate Adipose Tissue Energy Homeostasis and Inflammation. Cell Metab (2018) 27(2):378-92.e375. doi: 10.1016/j.cmet.2018.01.004

69. Perng W, Oken E, Dabelea D. Developmental Overnutrition and Obesity and Type 2 Diabetes in Offspring. Diabetologia (2019) 62(10):1779-88. doi: 10.1007/s00125-019-4914-1

70. Notarangelo FM, Beggiato S, Schwarcz R. Assessment of Prenatal Kynurenine Metabolism Using Tissue Slices: Focus on the Neosynthesis of Kynurenic Acid in Mice. Dev Neurosci (2019) 41(1-2):102-11. doi: 10.1159/ 000499736

71. Goeden N, Notarangelo FM, Pocivavsek A, Beggiato S, Bonnin A, Schwarcz R. Prenatal Dynamics of Kynurenine Pathway Metabolism in Mice: Focus on Kynurenic Acid. Dev Neurosci (2017) 39(6):519-28. doi: 10.1159/000481168

72. Michaelsen KF, Greer FR. Protein Needs Early in Life and Long-Term Health. Am J Clin Nutr (2014) 99(3):718S-22S. doi: 10.3945/ajcn.113.072603

73. Forbes JD, Azad MB, Vehling L, Tun HM, Konya TB, Guttman DS, et al. Association of Exposure to Formula in the Hospital and Subsequent Infant Feeding Practices With Gut Microbiota and Risk of Overweight in the First Year of Life. JAMA Pediatr (2018) 172(7):e181161. doi: 10.1001/ jamapediatrics.2018.1161

74. Dolecka J, Urbanik-Sypniewska T, Skrzydlo-Radomanska B, Parada-Turska J. Effect of Kynurenic Acid on the Viability of Probiotics In Vitro. Pharmacol Rep (2011) 63(2):548-51. doi: 10.1016/S1734-1140(11)70522-9 
75. Cheng H, Cheng Q, Bao X, Luo Y, Zhou Y, Li Y, et al. Over-Activation of NMDA Receptors Promotes ABCA1 Degradation and Foam Cell Formation. Biochim Biophys Acta Mol Cell Biol Lipids (2020) 1865 (10):158778. doi: 10.1016/j.bbalip.2020.158778

76. Farooq A, Hoque R, Ouyang X, Farooq A, Ghani A, Ahsan K, et al. Activation of N-Methyl-D-Aspartate Receptor Downregulates Inflammasome Activity and Liver Inflammation via a Beta-Arrestin-2 Pathway. Am J Physiol Gastrointest Liver Physiol (2014) 307(7):G732-740. doi: 10.1152/ajpgi.00073.2014

77. Prescott C, Weeks AM, Staley KJ, Partin KM. Kynurenic Acid has a Dual Action on AMPA Receptor Responses. Neurosci Lett (2006) 402(1-2):10812. doi: 10.1016/j.neulet.2006.03.051

78. Bertolino M, Vicini S, Costa E. Kynurenic Acid Inhibits the Activation of Kainic and N-Methyl-D-Aspartic Acid-Sensitive Ionotropic Receptors by a Different Mechanism. Neuropharmacology (1989) 28(5):453-7. doi: 10.1016/ 0028-3908(89)90078-6

79. O'Dowd BF, Nguyen T, Marchese A, Cheng R, Lynch KR, Heng HH, et al. Discovery of Three Novel G-Protein-Coupled Receptor Genes. Genomics (1998) 47(2):310-3. doi: 10.1006/geno.1998.5095

80. Wang J, Simonavicius N, Wu X, Swaminath G, Reagan J, Tian H, et al. Kynurenic Acid as a Ligand for Orphan G Protein-Coupled Receptor GPR35. J Biol Chem (2006) 281(31):22021-8. doi: 10.1074/jbc.M603503200

81. Jenkins L, Brea J, Smith NJ, Hudson BD, Reilly G, Bryant NJ, et al. Identification of Novel Species-Selective Agonists of the G-Protein-Coupled Receptor GPR35 That Promote Recruitment of Beta-Arrestin-2 and Activate Galpha13. Biochem $J$ (2010) 432(3):451-9. doi: 10.1042/BJ20101287

82. Mackenzie AE, Lappin JE, Taylor DL, Nicklin SA, Milligan G. GPR35 as a Novel Therapeutic Target. Front Endocrinol (Lausanne) (2011) 2:68. doi: 10.3389/fendo.2011.00068

83. Barth MC, Ahluwalia N, Anderson TJ, Hardy GJ, Sinha S, Alvarez-Cardona JA, et al. Kynurenic Acid Triggers Firm Arrest of Leukocytes to Vascular Endothelium Under Flow Conditions. J Biol Chem (2009) 284(29):19189-95. doi: 10.1074/jbc.M109.024042

84. Zheng X, Hu M, Zang X, Fan Q, Liu Y, Che Y, et al. Kynurenic Acid/GPR35 Axis Restricts NLRP3 Inflammasome Activation and Exacerbates Colitis in Mice With Social Stress. Brain Behav Immun (2019) 79:244-55. doi: 10.1016/j.bbi.2019.02.009

85. Cosi C, Mannaioni G, Cozzi A, Carla V, Sili M, Cavone L, et al. G-Protein Coupled Receptor 35 (GPR35) Activation and Inflammatory Pain: Studies on the Antinociceptive Effects of Kynurenic Acid and Zaprinast. Neuropharmacology (2011) 60(7-8):1227-31. doi: 10.1016/j.neuropharm.2010.11.014

86. Yang Y, Lu JY, Wu X, Summer S, Whoriskey J, Saris C, et al. G-Protein-Coupled Receptor 35 Is a Target of the Asthma Drugs Cromolyn Disodium and Nedocromil Sodium. Pharmacology (2010) 86(1):1-5. doi: 10.1159/000314164

87. Jung TW, Park J, Sun JL, Ahn SH, Abd El-Aty AM, Hacimuftuoglu A, et al. Administration of Kynurenic Acid Reduces Hyperlipidemia-Induced Inflammation and Insulin Resistance in Skeletal Muscle and Adipocytes. Mol Cell Endocrinol (2020) 518:110928. doi: 10.1016/j.mce.2020.110928

88. Beamer CA, Seaver BP, Shepherd DM. Aryl Hydrocarbon Receptor (AhR) Regulates Silica-Induced Inflammation But Not Fibrosis. Toxicol Sci (2012) 126(2):554-68. doi: 10.1093/toxsci/kfs024

89. DiNatale BC, Murray IA, Schroeder JC, Flaveny CA, Lahoti TS, Laurenzana EM, et al. Kynurenic Acid is a Potent Endogenous Aryl Hydrocarbon Receptor Ligand That Synergistically Induces Interleukin-6 in the Presence of Inflammatory Signaling. Toxicol Sci (2010) 115(1):89-97. doi: 10.1093/toxsci/kfq024

90. Hilmas C, Pereira EF, Alkondon M, Rassoulpour A, Schwarcz R, Albuquerque EX. The Brain Metabolite Kynurenic Acid Inhibits Alpha7 Nicotinic Receptor Activity and Increases non-Alpha7 Nicotinic Receptor Expression: Physiopathological Implications. J Neurosci (2001) 21 (19):7463-73. doi: 10.1523/JNEUROSCI.21-19-07463.2001

91. Brody SL, Wrenn KD, Wilber MM, Slovis CM. Predicting the Severity of Cocaine-Associated Rhabdomyolysis. Ann Emerg Med (1990) 19(10):113743. doi: 10.1016/S0196-0644(05)81518-5

92. Lugo-Huitron R, Blanco-Ayala T, Ugalde-Muniz P, Carrillo-Mora P, Pedraza-Chaverri J, Silva-Adaya D, et al. On the Antioxidant Properties of Kynurenic Acid: Free Radical Scavenging Activity and Inhibition of Oxidative Stress. Neurotoxicol Teratol (2011) 33(5):538-47. doi: 10.1016/ j.ntt.2011.07.002
93. Zhang P, Li T, Wu X, Nice EC, Huang C, Zhang Y. Oxidative Stress and Diabetes: Antioxidative Strategies. Front Med (2020) 14(5):583-600. doi: 10.1007/s11684-019-0729-1

94. Coto-Montes A, Boga JA, Tomas-Zapico C, Rodriguez-Colunga MJ, MartinezFraga J, Tolivia-Cadrecha D, et al. Physiological Oxidative Stress Model: Syrian Hamster Harderian Gland-Sex Differences in Antioxidant Enzymes. Free Radic Biol Med (2001) 30(7):785-92. doi: 10.1016/S0891-5849(01)00468-3

95. Csillik A, Knyihar E, Okuno E, Krisztin-Peva B, Csillik B, Vecsei L. Effect of 3-Nitropropionic Acid on Kynurenine Aminotransferase in the Rat Brain. Exp Neurol (2002) 177(1):233-41. doi: 10.1006/exnr.2002.7973

96. Luchowski P, Luchowska E, Turski WA, Urbanska EM. 1-Methyl-4Phenylpyridinium and 3-Nitropropionic Acid Diminish Cortical Synthesis of Kynurenic Acid via Interference With Kynurenine Aminotransferases in Rats. Neurosci Lett (2002) 330(1):49-52. doi: 10.1016/S0304-3940(02)00735-8

97. Castro-Portuguez R, Sutphin GL. Kynurenine Pathway, NAD(+) Synthesis, and Mitochondrial Function: Targeting Tryptophan Metabolism to Promote Longevity and Healthspan. Exp Gerontol (2020) 132:110841. doi: 10.1016/ j.exger.2020.110841

98. Thompson Legault J, Strittmatter L, Tardif J, Sharma R, TremblayVaillancourt V, Aubut C, et al. A Metabolic Signature of Mitochondrial Dysfunction Revealed Through a Monogenic Form of Leigh Syndrome. Cell Rep (2015) 13(5):981-9. doi: 10.1016/j.celrep.2015.09.054

99. Buchanan RW. The Effects of Kynurenine Aminotransferase Inhibition in People With Schizophrenia (TrypNAC-Ii). ClinicalTrials.gov. Maryland: University of Maryland (2019). NCT04013555.

100. Lindquist C, Bjorndal B, Lund A, Slettom G, Skorve J, Nygard O, et al. Increased Fatty Acid Oxidation and Mitochondrial Proliferation in Liver are Associated With Increased Plasma Kynurenine Metabolites and Nicotinamide Levels in Normolipidemic and Carnitine-Depleted Rats. Biochim Biophys Acta Mol Cell Biol Lipids (2020) 1865(2):158543. doi: 10.1016/j.bbalip.2019.158543

101. Hardeland R, Zsizsik BK, Poeggeler B, Fuhrberg B, Holst S, Coto-Montes A. Indole-3-Pyruvic and -Propionic Acids, Kynurenic Acid, and Related Metabolites as Luminophores and Free-Radical Scavengers. Adv Exp Med Biol (1999) 467:389-95. doi: 10.1007/978-1-4615-4709-9_49

102. Colin-Gonzalez AL, Paz-Loyola AL, de Lima ME, Galvan-Arzate S, Seminotti B, Ribeiro CA, et al. Experimental Evidence That 3Methylglutaric Acid Disturbs Mitochondrial Function and Induced Oxidative Stress in Rat Brain Synaptosomes: New Converging Mechanisms. Neurochem Res (2016) 41(10):2619-26. doi: 10.1007/s11064016-1973-2

103. Kessler M, Terramani T, Lynch G, Baudry M. A Glycine Site Associated With N-Methyl-D-Aspartic Acid Receptors: Characterization and Identification of a New Class of Antagonists. J Neurochem (1989) 52(4):1319-28. doi: 10.1111/j.1471-4159.1989.tb01881.x

104. Fukuda A, Muramatsu K, Okabe A, Shimano Y, Hida H, Fujimoto I, et al. NMDA Receptor-Mediated Differential Laminar Susceptibility to the Intracellular Ca2+ Accumulation Induced by Oxygen-Glucose Deprivation in Rat Neocortical Slices. J Neurophysiol (1998) 79(1):430-8. doi: 10.1152/ jn.1998.79.1.430

105. Baran H, Staniek K, Kepplinger B, Gille L, Stolze K, Nohl H. Kynurenic Acid Influences the Respiratory Parameters of Rat Heart Mitochondria. Pharmacology (2001) 62(2):119-23. doi: 10.1159/000056082

106. Baran H, Staniek K, Bertignol-Sporr M, Attam M, Kronsteiner C, Kepplinger B. Effects of Various Kynurenine Metabolites on Respiratory Parameters of Rat Brain, Liver and Heart Mitochondria. Int J Tryptophan Res (2016) 9:1729. doi: $10.4137 /$ IJTR.S37973

107. Guillemin GJ, Smith DG, Smythe GA, Armati PJ, Brew BJ. Expression of the Kynurenine Pathway Enzymes in Human Microglia and Macrophages. Adv Exp Med Biol (2003) 527:105-12. doi: 10.1007/978-1-4615-0135-0_12

Conflict of Interest: The authors declare that the research was conducted in the absence of any commercial or financial relationships that could be construed as a potential conflict of interest.

Publisher's Note: All claims expressed in this article are solely those of the authors and do not necessarily represent those of their affiliated organizations, or those of the publisher, the editors and the reviewers. Any product that may be evaluated in 
this article, or claim that may be made by its manufacturer, is not guaranteed or endorsed by the publisher.

Copyright $\odot 2022$ Zhen, Liu, Zhang and Song. This is an open-access article distributed under the terms of the Creative Commons Attribution License
(CC BY). The use, distribution or reproduction in other forums is permitted, provided the original author(s) and the copyright owner(s) are credited and that the original publication in this journal is cited, in accordance with accepted academic practice. No use, distribution or reproduction is permitted which does not comply with these terms. 